\title{
Hybrid digital-optical imaging design for reducing surface asphericity cost while keeping high performance
}

\author{
Javier Portilla, Sergio Barbero \\ Instituto de Optica (IO, CSIC), Madrid, Spain.
}

\begin{abstract}
Aspheres in optical design are frequently used to achieve high optical performance in imaging applications. However, manufacturing aspheres involves serious precision and cost issues that must be considered. We show that, by applying a hybrid digital-optical design approach, the amount of the asphericity cost in a single surface may be significantly alleviated without compromising the performance. First, we compute the amount of spherical aberration depending on the optical parameters (conic constant and shape factor), and compute its corresponding point spread function (PSF). From the PSF and the spectral distribution of clean images and noise, we set a statistical observation model for estimating the expected image quality (in mean square error terms) in the image sensor and also in the digitally restored image. In addition, we use a previously proposed metric for quantifying the asphere fabrication cost, to set different cost scenarios. For each of these scenarios, we study how image quality is optimized, before and after digital restoration. Reversely, we find the optical configurations of minimal asphericity cost amongst those providing a very low aberration level. Although here we have limited our study to just on-axis, monochromatic imaging, we show in simulations how our digital-optical combined approach has a high potential for boosting the cost-effectiveness trade-off.
\end{abstract}

Keywords: Hybrid digital-optical design; Asphere optics; Lens system design; Digital image restoration; Cost-effectiveness analysis in optical-digital design

\section{INTRODUCTION}

The use of aspherical surfaces is nowadays a common practice in a wide range of imaging applications. Theoretically, an aspheric surface may have an arbitrary number of shape parameters (e.g. using a piecewise polynomial representation), thus providing additional design degrees of freedom with respect to a spherical surface. In practice, most designs use aspheres mathematically represented by a fixed number of parameters.

Aspheres in optical design are frequently used to achieve high optical performance, especially in those systems dominated by aperture-dependent aberrations. ${ }^{1}$ Also, given a prescribed imaging performance, an optical system involving asphericities would require less number of elements than a spherical one. However, manufacturing aspheres, and subsequently metrological testing of such surfaces, involves serious precision and cost issues that must be considered when designing commercially viable systems. ${ }^{2}$ Indeed, this has lead to new mathematical representations of optical aspheres that intrinsically take into account these issues. ${ }^{3-5}$ Therefore, imaging performance improvement, on the one hand, and manufacturing precision and cost constraints, on the other hand, pose a relevant trade-off problem in optical design with aspheres. To get a deeper insight into this trade-off one may pose the question: How much performance improvement is achieved by the use of asphericity with respect to a corresponding spherical design? Schulz made a serious attempt, exclusively guided by optical criteria, to develop a theoretical framework providing quantitative answers to this question. ${ }^{6}$ However, nowadays many optical systems are accompanied by a (possibly embedded) digital image processing step that greatly improves the final digital image quality (DQ). Moreover, the emerging field of hybrid digitaloptical design, through optimizing metrics that predict the final DQ, jointly considers the optical and the digital processing steps. ${ }^{7,8}$ Our goal here is to show that, by applying a cost-effectiveness, hybrid digital-optical design approach, the nominal cost (evaluated through a simple model) associated to asphericity on a single surface may be significantly alleviated, without compromising the final performance.

This work was supported by grants FIS2016-75891-P (Spanish Ministerio de Economia, Industria y Competitividad). 


\section{METHODS}

Here we start by exploring one of the simplest lens-based structure involving asphericity: a thin lens comprising an anterior spherical surface and a posterior conic one. Although such system introduces noticeable off-axis and chromatic aberration, here we will just consider on-axis quality under monochromatic light.

\subsection{Optical characterization}

The general procedure followed here comprises two steps: (1) to compute the wave aberration function from the optical parameters; (2) to compute the Point Spread Function through the Fourier transform assuming far-field diffraction approximation.

For the above described system, the wave aberration function, within third-order aberration theory, just have one spherical aberration term, $A_{s}$ :

$$
W(r, \theta)=A_{s} r^{4} .
$$

Through primary aberration theory, $A_{s}$ can be approximated as an analytical function of the involved optical parameters, by: ${ }^{1}$

$$
A_{s}=\frac{(n-1) Q}{8 R_{p}^{3}}-\frac{1}{32 n(n-1) f^{3}}\left(\frac{n^{3}}{n-1}+(3 n+2)(n-1) P_{f}^{2}+\frac{(n+2) S_{f}^{2}}{n-1}+4(n+1) P_{f} S_{f}\right) .
$$

$R_{a}$ and $R_{p}$ denote the anterior and posterior radii of curvature, respectively. $Q$ denotes the posterior conic constant. Besides, $f$ is the focal length, $n$ the refractive index, and $P_{f}$ and $S_{f}$ are the Coddington position and shape factors, respectively:

$$
P_{f}=\frac{s^{\prime}+s}{s^{\prime}-s}, \quad S_{f}=\frac{R_{a}+R_{p}}{R_{p}-R_{a}},
$$

where $s$ and $s^{\prime}$ are the object and image planes distances to the thin lens plane, respectively.

Within Fraunhofer diffraction theory, ${ }^{9}$ the PSFs $h\left(x^{\prime}, y^{\prime} ; A_{s}\right)$ can be approximated from $W(x, y)$ by using the Fourier Transform $(\mathcal{F}\{\})$ :

$$
h\left(x^{\prime}, y^{\prime}\right)=|\mathcal{F}\{A(\hat{x}, \hat{y}) \exp (-i 2 \pi W(\hat{x}, \hat{y}))\}|^{2},
$$

where $A$ is a uniform radiance pupil transmittance function, and $\hat{x}, \hat{y}=(x, y) /(\lambda f)$, with $f$ the focal length and $\lambda$ the wavelength.

\subsection{Mean Square Error prediction}

Hybrid digital-optical design assumes there is an image restoration stage applied to the sensor image, and, consequently, it requires to make a prediction about how changes in the optical parameters affect the image quality after restoration. In the simplest case (see, e.g. ${ }^{8}$ ), the image quality is measured through the expected mean square error (MSE), and, as pointed above, restoration is assumed to be linear and shift-invariant (Wiener regularized deconvolution). For comparison purposes, it is also convenient to predict the amount of error on the sensor, prior to restoration.

\subsubsection{Observation model}

In order to make a full evaluation of the quality corresponding to each optical configuration, we make some assumptions:

- An observation model describing the image at the output of the sensor. Since in this work we have constrained ourselves to on-axis quality, we will just consider a single PSF applied uniformly to the image. This must not be understood as a extended object (off-axis) model, but as a local approximation that will allows us to predict on-axis image quality through simple computations in the Fourier domain.

$$
\mathbf{y}=\mathbf{h} * \mathbf{x}+\mathbf{w} .
$$

The symbol "*" denotes convolution, $\mathbf{h}$ is the PSF, $\mathbf{x}$ is the ideal image, and $\mathbf{w}$ is the noise. 
- A power spectral density (PSD) model for typical images. We used $P_{x}(f)=k / f^{2}$, for $f \neq 0$ and 0 in the origin (see, e.g. ${ }^{10}$ ), where here $f=\|\mathbf{f}\|$ is the radial spatial frequency. $k$ is computed to satisfy that the variance (PSD's integral, excluding zero) is $\sigma_{x}^{2}=1$.

- A spectral model for the sensor's noise. For simplicity we assume white noise: $P_{w}=\sigma_{w}^{2}$. We consider three sensor noise scenarios: low, medium, and high, corresponding to $\sigma_{w} / \sigma_{x}=\{1 / 128,1 / 32,1 / 8\}$. If there was no optical blur, that would correspond to signal-to-noise ratios (SNR) in the sensor image of 42, 30 and $18 \mathrm{~dB}$, respectively. The lowest noise scenario corresponds approximately to a typical photography with good illumination (average of $18 \%$ of detector's full-well capacity) using a high-end consumer sensor. ${ }^{11}$

\subsubsection{MSE on the sensor}

A simple metric to quantify the amount of optical blur is the $R M S$ of the PSF, understood as the square root of PSF's second order moment. However, for quantitatively assessing the impact of the optical blur on the image quality we need to consider additional factors: the Optical Transfer Function full shape $(H(\mathbf{f}))$, and the Power Spectral Densities (PSDs) of both uncorrupted image $\left(P_{x}(\mathbf{f})\right)$ and noise $\left(P_{w}(\mathbf{f})\right)$. With this information, and under the model assumptions in section 2.2.1, it is straightforward to compute the expected MSE of the image on the sensor:

$$
M S E_{S}=\int_{f \neq 0} P_{e}^{(S)}(\mathbf{f}) d \mathbf{f}
$$

where $P_{e}^{(S)}(\mathbf{f})=\mathbb{E}\left\{|Y(\mathbf{f})-X(\mathbf{f})|^{2}\right\}$. Substituting $Y(\mathbf{f})=H(\mathbf{f}) X(\mathbf{f})+W(\mathbf{f})$ (Eq. 5 in Fourier domain) and operating, we obtain:

$$
\begin{aligned}
P_{e}^{(S)}(\mathbf{f}) & =P_{w}(\mathbf{f})+P_{x}(\mathbf{f})|1-H(\mathbf{f})|^{2} \\
& =\sigma^{2}+|1-H(\mathbf{f})|^{2} / f^{2} .
\end{aligned}
$$

\subsubsection{MSE after linear restoration (Wiener filter)}

Under a hybrid optical-digital design perspective, the image at the sensor's output is just an intermediate result. Although the result after applying a digital restoration method to the sensor image will depend on the details of the applied method, one can nevertheless take as a useful first approximation to the shape of the digital MSE landscape (as a function of the parameters being optimized), the much-simpler-to-compute MSE at the output of an optimal linear (Wiener) filter. ${ }^{8,12,13}$ Naturally, this approximation during the design stage does not preclude us from using a different, more powerful, non-linear restoration method in operation time (as illustrated here in section 3.4).We can write the expected MSE in this case by:

$$
M S E_{R}=\int_{f \neq 0} P_{e}^{(R)}(\mathbf{f}) d \mathbf{f}
$$

where, in our case, $P_{e}^{(R)}(\mathbf{f})=\mathbb{E}\left\{\left|H_{w}(\mathbf{f}) Y(\mathbf{f})-X(\mathbf{f})\right|^{2}\right\}$, and $H_{w}(\mathbf{f})=P_{x}(\mathbf{f}) H^{*}(\mathbf{f}) /\left(|H(\mathbf{f})|^{2} P_{x}(\mathbf{f})+P_{w}(\mathbf{f})\right)$ is the Wiener filter. Operating, we obtain:

$$
\begin{aligned}
P_{e}^{(R)}(\mathbf{f}) & =\frac{P_{x}(\mathbf{f}) P_{w}(\mathbf{f})}{P_{x}(\mathbf{f})|H(\mathbf{f})|^{2}+P_{w}(\mathbf{f})} \\
& =\left(|H(\mathbf{f})|^{2} / \sigma^{2}+f^{2}\right)^{-1} .
\end{aligned}
$$

\subsection{Manufacturing cost of aspheres}

In this work we restricted ourselves to rotationally symmetric conicoids for the aspherical surfaces; hence, two parameters: the radius of curvature $(R)$ on the vertex, and the conic constant $(Q)$ characterize the surface:

$$
z(r)=\frac{r^{2}}{R\left(1+\sqrt{1-(Q+1) r^{2} / R^{2}}\right)},
$$


where $\mathrm{r}$ is the radial coordinate. For our analysis, it is convenient to refer to a metric evaluating the manufacturability and metrological measurement cost of an asphere. From the different proposals (see, e.g., discussion on this issue $\mathrm{at}^{4}$ ), we chose a metric based on the deviation of the surface slope from that of a best-fit reference sphere ${ }^{2}$ termed Mean Slope Absolute Error (MSAE). It is defined as:

$$
M S A E=\int_{0}^{R_{\text {pupil }}}\left|\frac{z(r)}{d r}-\frac{z_{o}(r)}{d r}\right| d r,
$$

where $z_{o}(r)=r^{2} /\left(R_{f i t}\left(1+\sqrt{1-r^{2} / R_{f i t}^{2}}\right)\right)$. Note that MSAE increases with the absolute value of $Q$ and the vertex curvature $1 / R$.

\section{SIMULATIONS}

In our simulations we have chosen the following parameters, in values and ranges: Coddington position factor $P_{f}=-1$ (objects at infinity), refraction index $n=1.5$, pupil radius $R_{\text {pupil }}=2 \mathrm{~mm}$, focal length $f=50 \mathrm{~mm}$, wavelength $\lambda=500 \mathrm{~nm}$, conic constant $Q \in[-6,0]$, shape factor $S_{f} \in[-2,2]$ (with a particular example for $S_{f}=-1$ ), sampling on the sensor plane in a $N_{p} \times N_{p}$ grid, with $N_{p}=512$.

\subsection{How does spherical aberration depends on lens geometry?}

First thing to examine is the behavior of the spherical aberration as a function of the conic constant $Q$ and the shape factor $S_{f}, A_{s}\left(Q, S_{f}\right)$, see Figure1. It is noticeable how, for shape factors between 0 and 1 , the influence of the conic factor $Q$ is much weaker, due to the larger radius of the posterior lens aspherical surface. For higher shape factors $A_{s}$ is only slightly affected by $Q$.

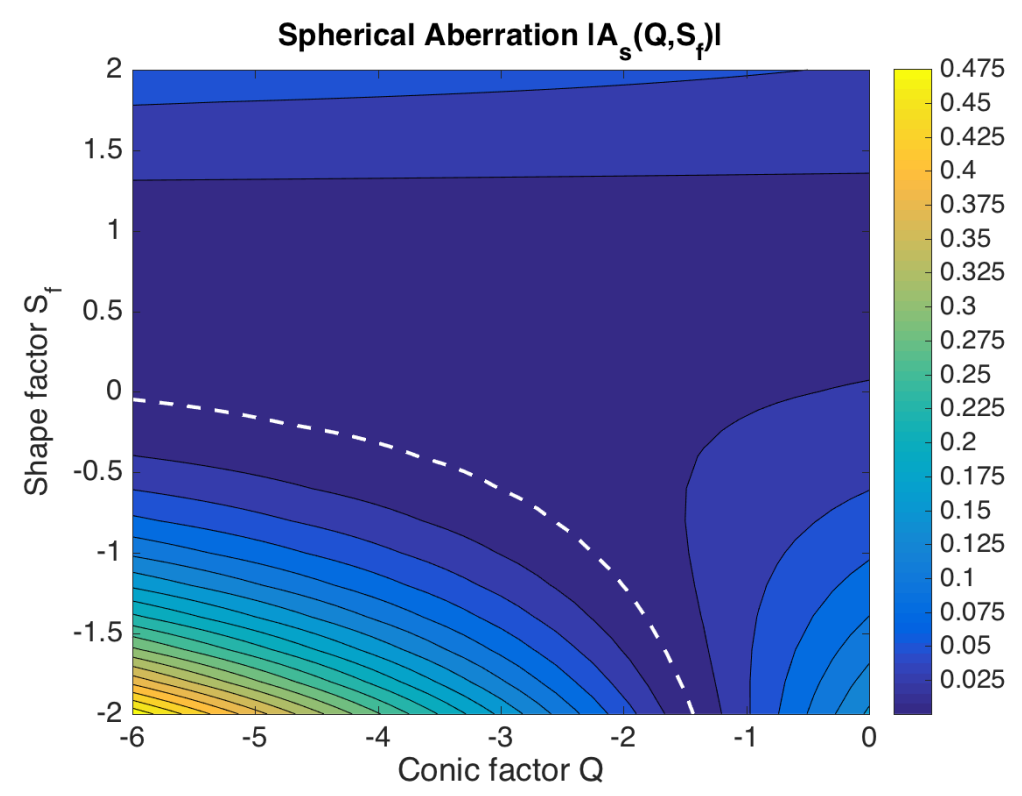

Figure 1. Modulus of the spherical aberration coefficient $\left|A_{s}\right|$ as function of conic constant $Q$ and shape factor $S_{f}$. The dashed white line indicates the locus of the aberration-free configurations.

\subsection{How does image quality depends on spherical aberration?}

Here we examine the effect of spherical aberration on different metrics of image quality. First, we consider the square root of the second order moment of the corresponding PSF, termed $R M S$. As we can see in Fig.2(a), except for a small correction close to zero (due to diffraction effects), this measurement does not depart much from $\left|A_{s}\right|$ itself. On Fig.2(b) we show the estimated MSE values, both for the sensor plane and 


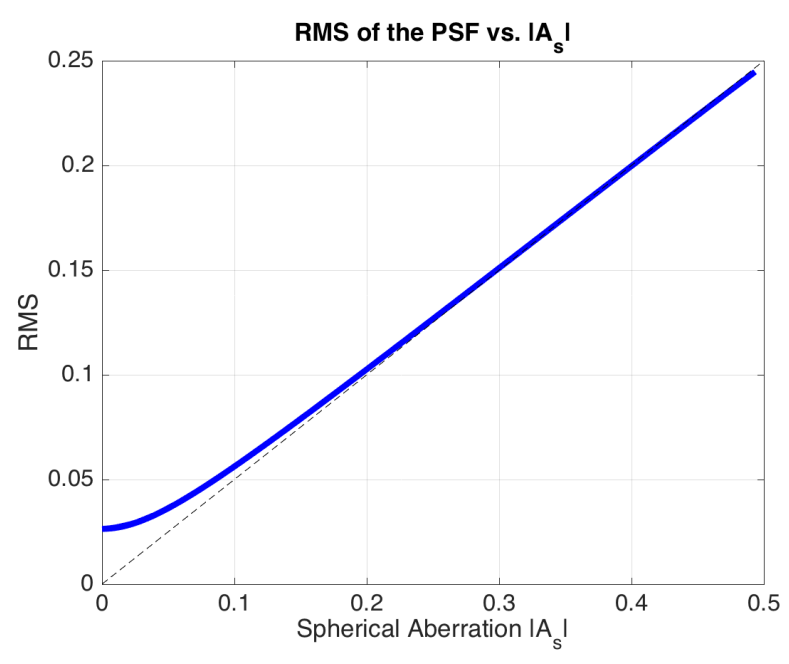

(a)

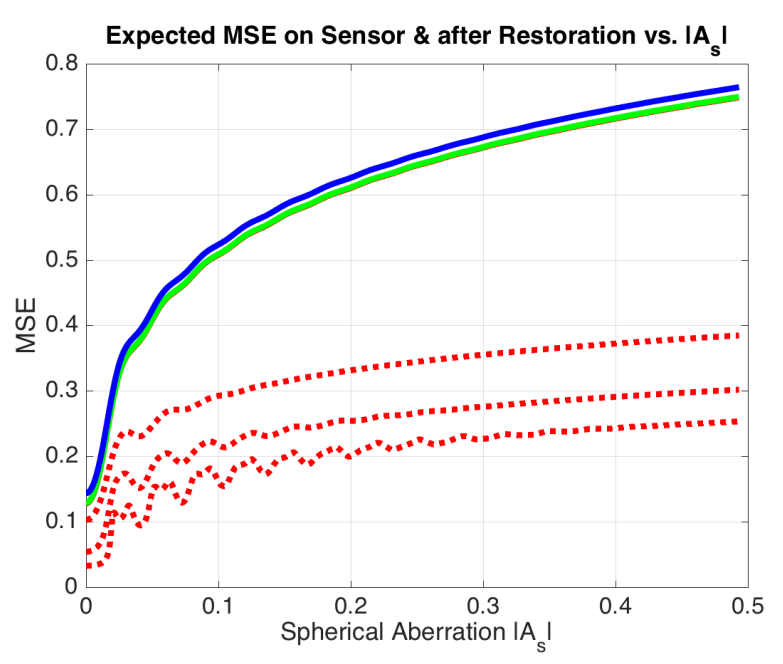

(b)

Figure 2. (a) RMS (square root of the PSF's second order moment) as a function of $\left|A_{s}\right|$. (b) Expected Mean Square Error, both at the sensor (continuous lines) and after Wiener restoration (dotted lines) for the three noise regimes considered, as a function of $\left|A_{s}\right|$.

after restoration, for the three noise levels considered. We see how the pure optical measurement ( $R M S$ of the PSF), in comparison with sensor's MSE estimate, is far from adequate, as it underestimates the impact of low aberration values, while overestimating it for high aberration values (this is in agreement with ${ }^{8}$ ). Furthermore, by observing the significantly lower slope of the dashed-line curves as compared to the continuous ones, we can conclude that digital restored images, in moderate to high aberration scenarios, are less sensitive to increments in spherical aberration in comparison to sensor images. In contrast, an important drop of the image quality occurs for relatively small aberration factors, for both sensor and restored images.

We can also see that, as the noise level decreases, ripples of the estimated MSE of the restored image as function of the spherical aberration become more and more important. Whereas, in absence of other cost considerations, a purely monotonic function will not have any effect on the location of the optimal configurations of the optical parameters, the existence of ripples breaking the monotonicity implies that small reductions of $A_{s}$ will not always result in a DQ improvement. More importantly, in this paper we consider an additional cost function, namely, one reflecting the cost associated to fabricating and testing aspheres (as explained in section 2.3). Under such multi-cost scenario, if there is an overall merit function, almost any change of one of the involved cost functions (e.g., by introducing or improving digital restoration, or by reducing the fabrication cost) will have an effect on the optima locations of the overall criterion (see discussion in section4).

\subsection{Optical and Digital Relative Gains}

Now we aim at quantifying the improvement we may expect from using asphericity as compared to that of including a digital restoration stage, either as a substitute for asphericity, or in addition to it. For that purpose, we define Optical Relative Gain (ORG) as the MSE reduction factor caused by using an aspherical lens surface of a given conic constant $Q$, with respect to using a purely spherical lens: $O R G(Q)=M S E_{S}(0) / M S E_{S}(Q)$. Analogously, we define Digital Relative Gain as $D R G(Q)=M S E_{S}(0) / M S E_{R}(Q)$. A special case corresponds to using digital image restoration without asphericity, which we characterize by $D R G_{0}=D R G(0)$.

In Figure 3(a) we show ORG and DRG for optimal $Q$, for each shape factor $S_{f}$, for the three considered noise levels. As we show in the section 3.4, the Wiener expected MSE is a very conservative guess for the real MSE when using competitive (state-of-the-art, or close) non-linear restoration. Even so, DRG gains (dotted lines) are much higher than ORG ones (solid line). For the $Q=0$ case, the digital relative gain $D R G_{0}$ (dashed line) is often comparable (or even superior) to ORG. Note that ORG does not depend much on the noise level, and thus it appears as a single line, whereas DRG is very sensitive to noise. Figure 3(b) shows the ORG and 


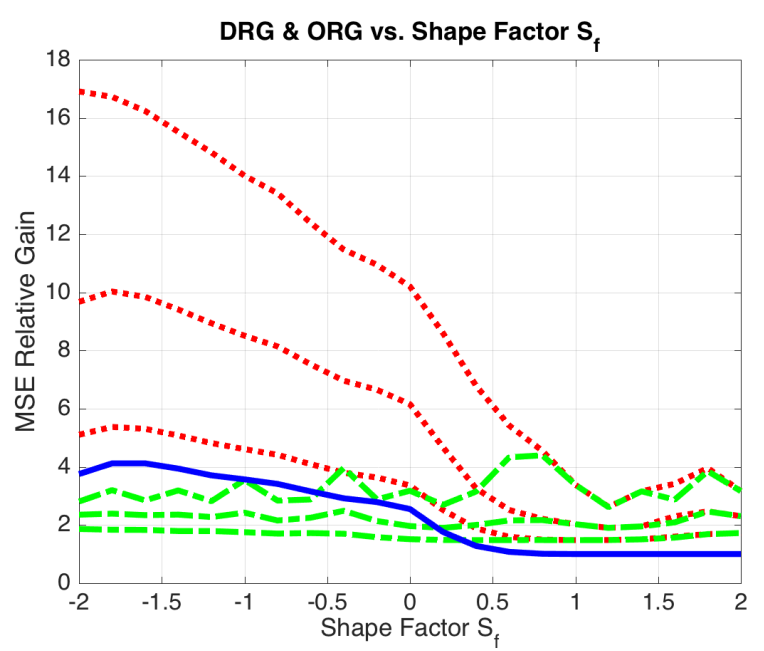

(a)

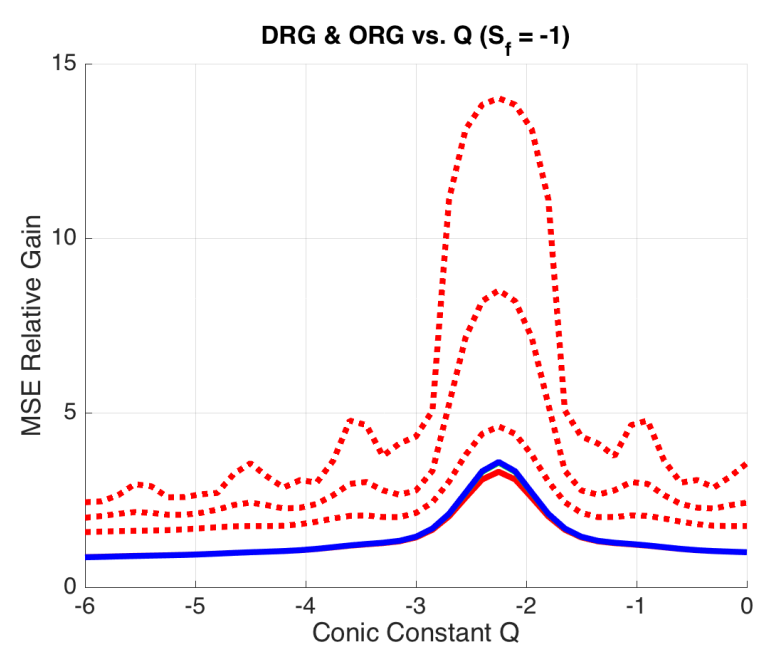

(b)

Figure 3. (a) Optimal Q relative gains, for ORG (solid line), DRG (dotted line) and DRG(0) (dashed line), as a function of the shape factor $S_{f}$, and for the three considered noise levels. (b) ORG (solid line) and DRG (dotted line) as a function of the conic constant $Q$, for the three considered noise levels, and for the shape factor $S_{f}=-1$.

DRG plots as a function of $Q$, for the particular case of $S_{f}=-1$ (plano-convex lens). We observe: (1) The DRG with no asphericity was roughly equal to the ORG with optimal asphericity (3.55 vs. 3.59, respectively); (2) at optimal asphericity DRG was 4 times higher than ORG (14.3 vs. 3.59).

\subsection{Some visual results}

Here we provide some visual examples, by applying a simple shift invariant PSF model. Figure 4 shows some results for the $S_{f}=-1, \mathrm{SNR}=42 \mathrm{~dB}$, case. Panel (a) shows simulated image crops for $Q=0$, for the sensor image (left), after Wiener restoration (center), and after applying $L 2-r-L O$, a recent non-linear restoration method of ours based on sparse image representation. ${ }^{14}$ Measured SNR values are: $6.34 \mathrm{~dB}$ (sensor), $14.30 \mathrm{~dB}$ (Wiener), and $16.49 \mathrm{~dB}$ (L2-r-L0). Panel (b) shows the corresponding results for the optimal conic constant, $Q=-2.25$. SNR values in this case are: $15.84 \mathrm{~dB}$ (sensor), $21.28 \mathrm{~dB}$ (Wiener), and $23.79 \mathrm{~dB}$ (L2-r-L0).

\subsection{Mean Slope Absolute Error as an indicator of fabrication costs}

We have seen how there are different combinations of conic constant and shape factor cancelling spherical aberration. However, not all of them are equivalent in terms of fabrication effort. As pointed out in Section 2.3, according to the MSAE metric, the fabrication cost grows both with the absolute value of the amount of asphericity (conic constant $Q$ ) and with the vertex curvature $1 / R$. As a consequence, it results in a non-trivial dependency on $Q$ and the shape factor $S_{f}$, as shown in Figure 5(a). Figure 5(b) displays the MSAE index for the zero-aberration solutions marked as a white line in panel (a). The conclusion is surprising: less costly solutions are, in this case, those with a high absolute conic factor and close to zero shape factor. This can be explained because the vertex curvature quickly decays when going from right to left (white line curve, in Figure 5(a)) from strong negative shape factors to a near bi-convex lens, and this has a stronger effect on the MSAE cost than the increasing of the conic constant $Q$.

A different way to extract useful information from the MSAE consists of fixing some MSAE levels, and evaluating the image quality along those curves. That allows us for studying the optimal configuration for a given fabrication cost. In Figure 6 we have displayed a contour color representations of the Mean Square Error on the sensor (panel (a)) and after restoration (panel (b)), for the low noise level case, along with those (white, dashed) level curves for MSAE values of $10^{-3}, 2.5 \times 10^{-4}$ and $6.3 \times 10^{-5}$. Finally, in Figure 7 we show the corresponding image quality, on sensor and after restoration (for the three noise level considered), measured as Signal-to-Noise ratio, in decibels. It is worth to note a number of features in these graphs: (1) 


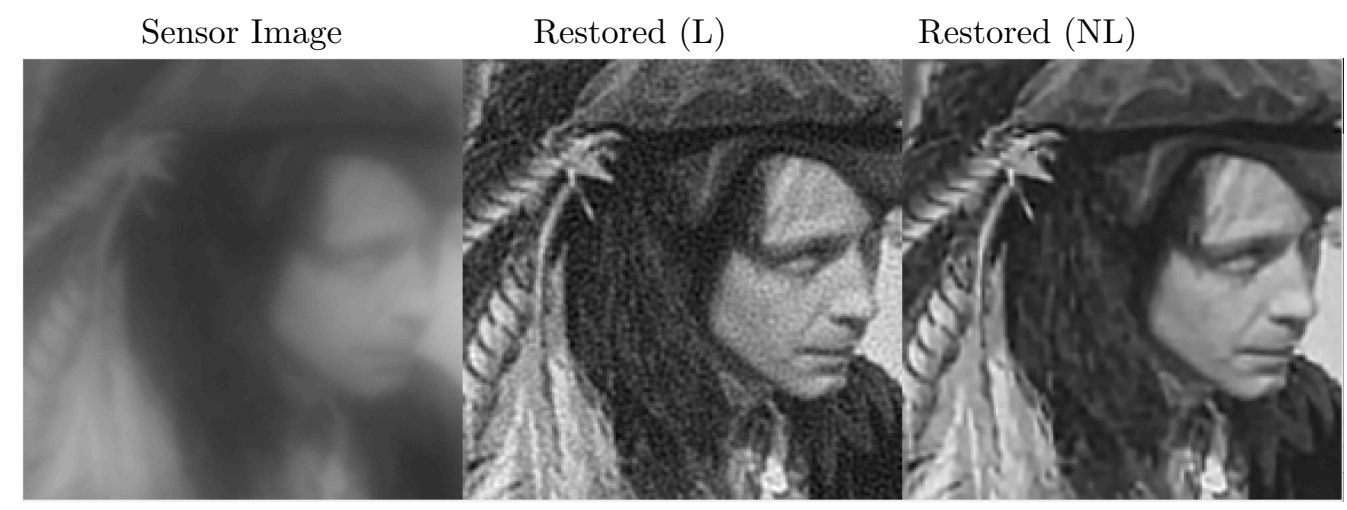

(a)

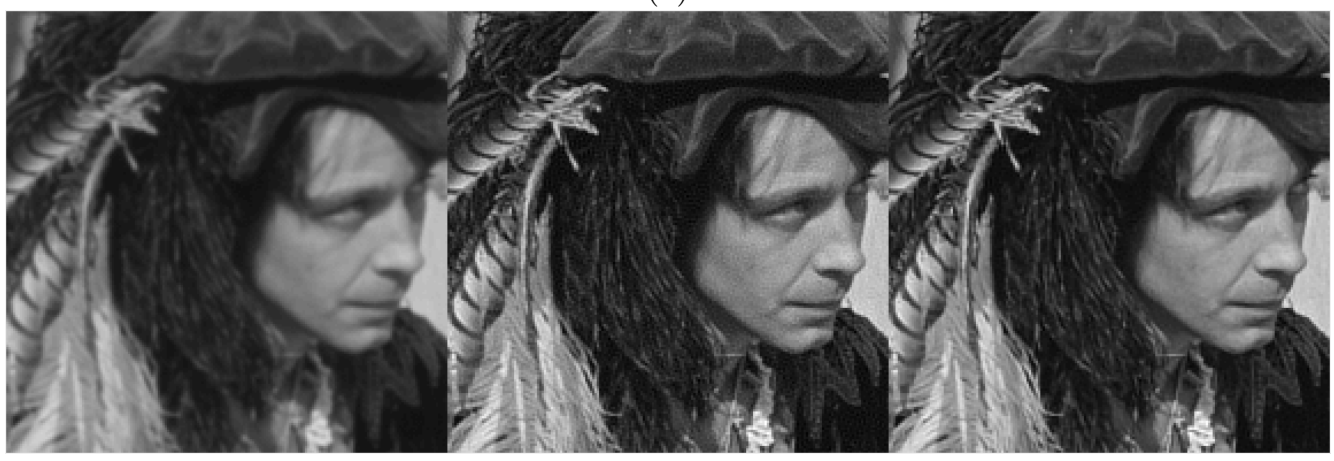

(b)

Figure 4. Simulated observation crops: sensor (left), results with Wiener (center) and $L 2-r-L O^{14}$ (right), for $\sigma_{w}=1 / 128$ (low noise regime). Panel (a): $Q=0$, panel (b): optimal Q $(Q=-2.25)$.

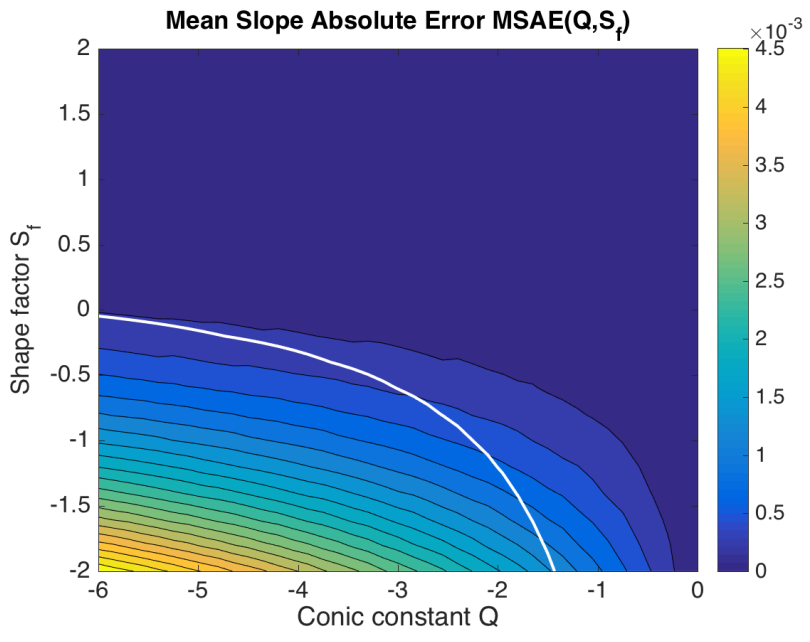

(a)

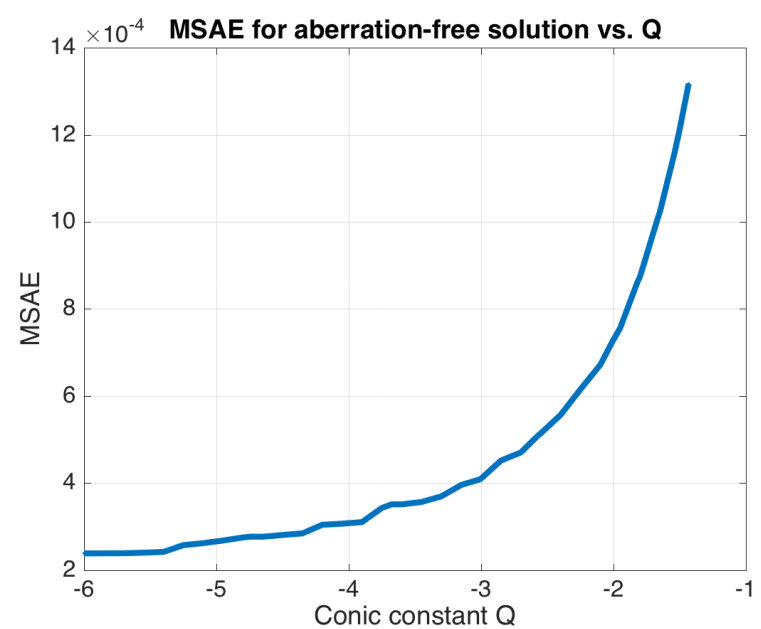

(b)

Figure 5. (a) MSAE index of the fabrication effort, as a function of the conic constant $Q$ and the shape factor $S_{f}$. (b) Evaluating MSAE for the aberration-free white curve of panel (a). 


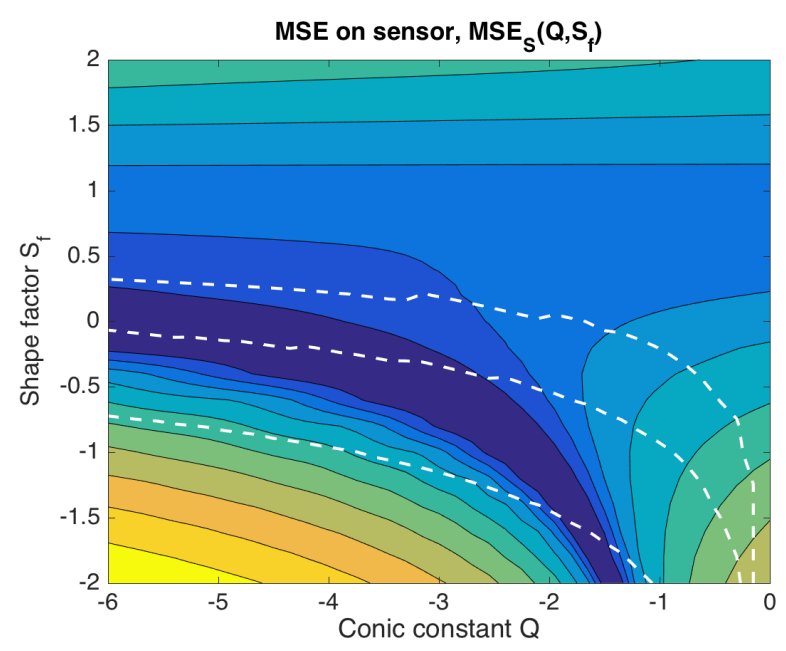

(a)

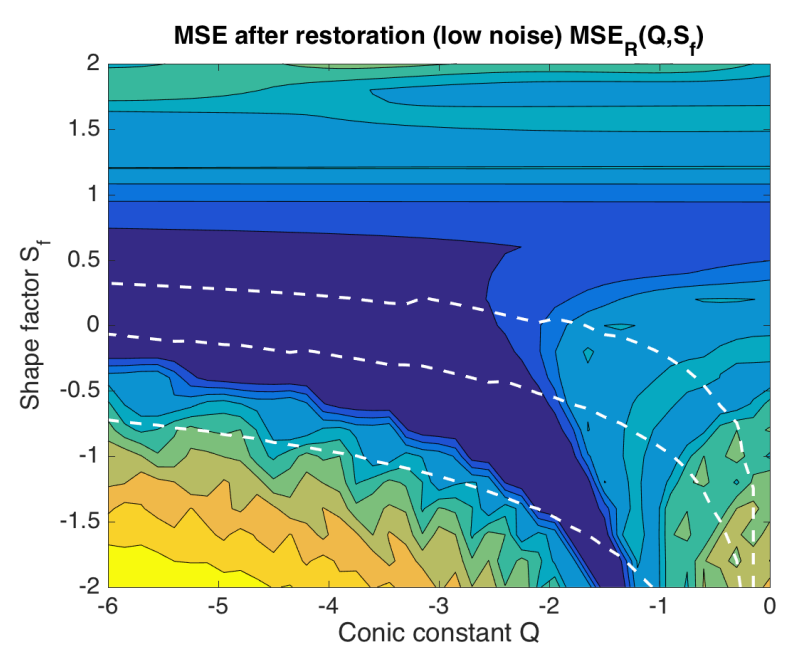

(b)

Figure 6. Mean square error on the sensor (panel (a)) and after restoration (panel (b), for low noise level). The dashed white lines correspond to three different MSAE levels: $10^{-3}, 2.5 \times 10^{-4}$ and $6.3 \times 10^{-5}$ (bottom-up)

Sensor and Restoration SNR shapes get gradually more and more similar as the noise level increases; (2) Higher MSAE levels do not necessarily provide lower MSE results; (3) Expected SNR after restoration is affected by oscillations, which tend to grow in amplitud as noise level decreases; (4) In the low-noise restoration scenario, difference in performance due to relatively large aberration gaps is sensibly narrowed, with respect to sensor and not-so-small-noise cases. This is just a consequence of the slow slope of the dotted curves in Figure 2(b), for high aberration values. In addition, comparison between high and medium MSAE curves provides a clear winner, namely, the medium MSAE level: while being less costly, it nevertheless provides a similar performance peak in all studied cases. On the other hand, for the medium and high noise scenarios, the large performance gap between the low and the middle MSAE levels will typically advice for choosing the medium MSAE level, again. The low noise restoration case is a little different, though: relatively small differences in SNR are found now between the medium and low MSAE level curves, for high absolute conic constants. Therefore, in this case, depending on the overall relative importance of the fabrication cost vs. the image quality for each design scenario, any of the two options could be chosen.

\section{CONCLUSIONS}

We have shown how cost-effectiveness associated to asphericity in imaging systems can be boosted by means of including a digital restoration stage in the design. Non-trivial dependency between the amount of asphericity and other design parameters (such as the involved lenses' shape factors), together with the sensor signal-to-noise ratio operation regime, must be considered in order to optimize the quality for a given cost (or vice versa).

A relevant aspect to consider is the determinant role that the involved cost functions play in the design. In this regard we want to warn that MSAE metric may not be the best option for all applications. We used it here just as an illustrative example. Regarding the image quality metrics, the mean square error (MSE) was chosen not because of being the best visual metric (which clearly is not), but because is the simplest in terms of theoretical formulation and practical calculations. Something analogous happens with the linear error prediction on the digitally restored image. Whereas a more powerful non-linear method, according to actual non-linear restoration algorithms, would be desirable, the Wiener solution requires a much simpler formulation and calculation.

Secondly, whereas one can always carry on an iso-level approach (as the one we performed here) consisting on fixing one cost function and marginally optimizing the other, for a number of iso-level curves, an alternative, yet classical, strategy consists of defining an overall merit function involving simultaneously fabrication cost 


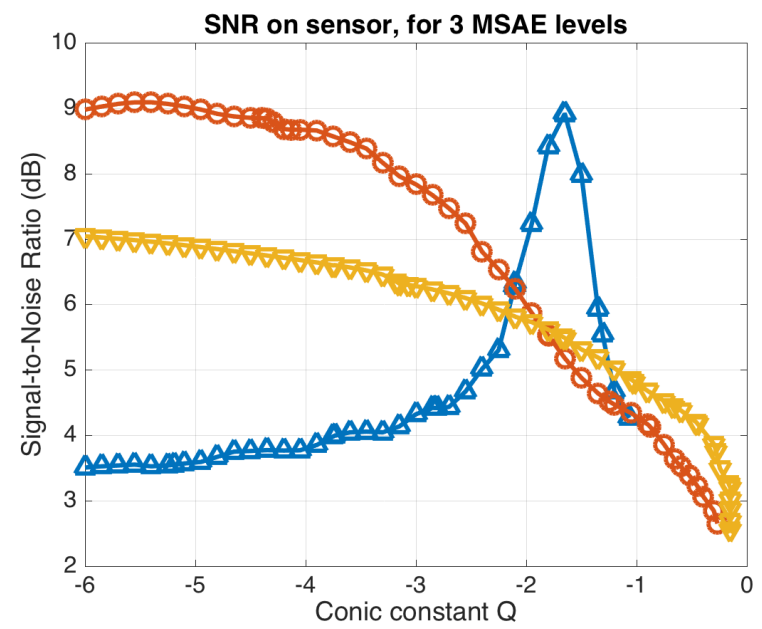

(a)

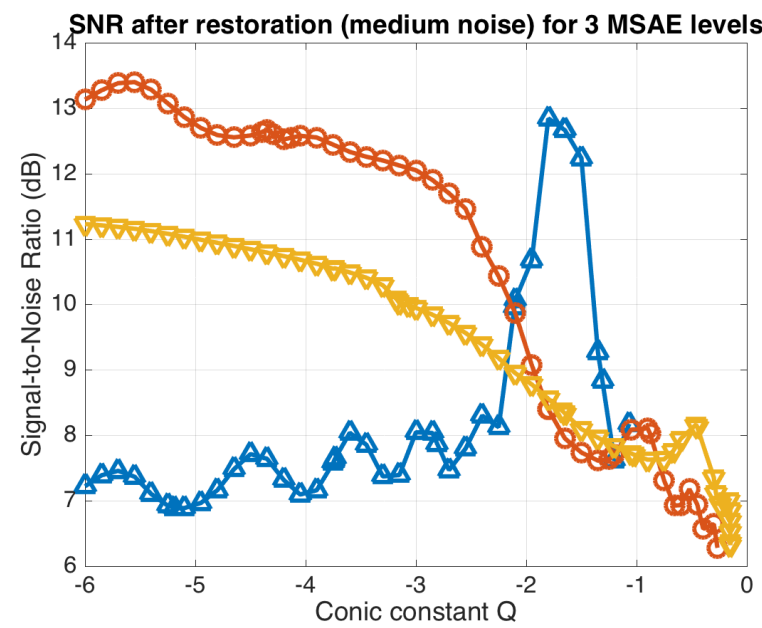

(c)

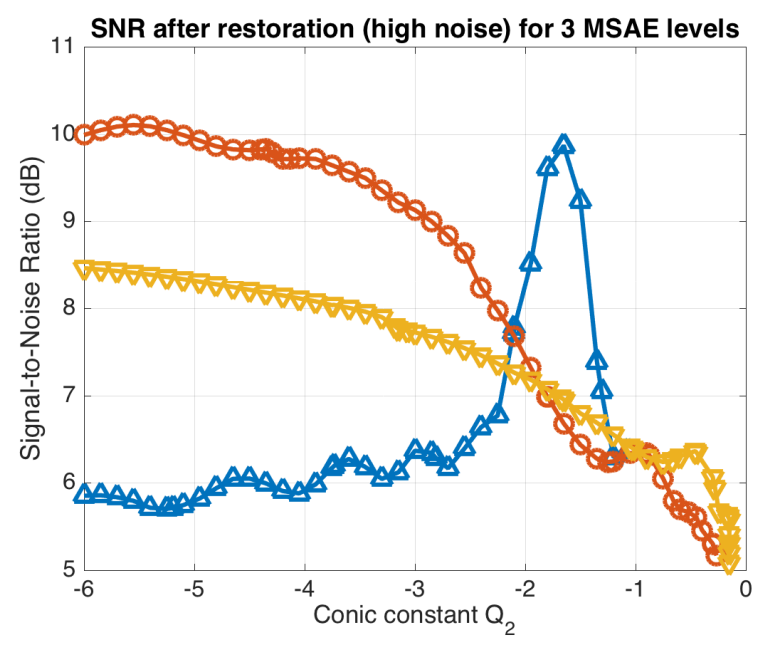

(b)

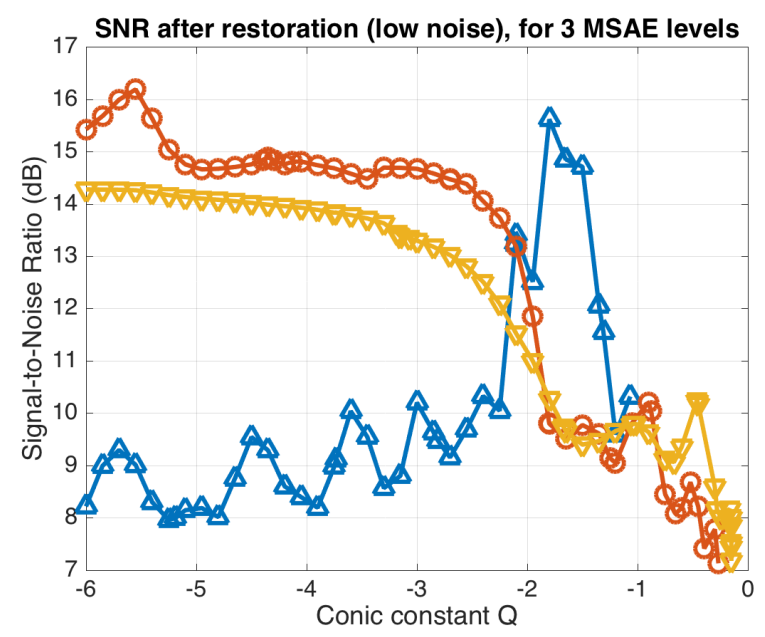

(d)

Figure 7. Evaluating the mean square error (expressed here as Signal-to-Noise Ratio, SNR) of the iso-MSAE curves depicted in Fig. 6, for the different scenarios. (a) Sensor error; (b), (c) and (d): Restoration error, for high, medium and low noise levels, respectively. High MSAE is indicated by up-triangles, medium MSAE by circles, and low MSAE by down-triangles. 
and expected performance. The particular form and relative weights of that merit function will, naturally, be highly dependent on the priorities and constraints set for the design. It is nevertheless worth noting that, when using a joint criterion, even monotonic functions individually applied to the involved merit/cost functions would generally have an effect on the location of the optima in the overall merit function. This is relevant for hybrid digital-optical design, because it implies that, even if the optimal-performance parameters remain almost unchanged when going from the sensor to the digitally restored image (as in the case presented in this paper), any significant difference between their corresponding merit functions (like those seen in Figure 2(b)) will impact the final design.

Finally, we note that, for the single-aberration case, all quality metrics are function of the corresponding aberration coefficient. Furthermore, if we disregard ripple-generating diffraction effects, all these functions are monotonic (whatever the metric is, it always gets worse when a single aberration increases), and, therefore, they all have the same optima locations. In the case studied here, diffraction produces just a modest deviation from that situation. In a richer scenario, though, with more than one aberration (e.g., off-axis and chromatic aberrations), the optical parameters optimizing the image quality on sensor vs. the restored image (and/or restored image for different noise regimes), will significantly depart from each other (as happened, e.g., in ${ }^{8}$ for the defocus-spherical aberration trade-off).

\section{REFERENCES}

[1] Gross, H., Blechinger, F., Peschka, M., Singer, W., and Zgge, H., [Handbook of Optical Systems: Aberration Theory and Correction of Optical Systems. Volume 3], John Wiley-Sons, Incorporated (2007).

[2] Kumler, J., "Designing and specifying aspheres for manufacturability," Proc. SPIE. Current Developments in Lens Design and Optical Engineering 5874, 1-9 (2005).

[3] Forbes, G., "Shape specification for axially symmetric optical surfaces," Opt. Express 15(8), 5218-5226 (2007).

[4] Forbes, G., "Manufacturability estimates for optical aspheres," Opt. Express 19(10), 9923-9942 (2011).

[5] Forbes, G., "Characterizing the shape of freeform optics," Opt. Express 20(3), 2483-2499 (2012).

[6] Schultz, G., "Imaging performance of aspherics in comparison with spherical surfaces," Appl. Opt. 26(23), 5118-5124 (1987).

[7] Stork, D. G. and Robinson, M. D., "Theoretical foundations for joint digital-optical analysis of electrooptical imaging systems," Appl. Opt. 47(10), B64-B75 (2008).

[8] Portilla, J. and Barbero, S., "Comparing optical to digital metrics: What is the optimal defocus in a rotationally symmetric system?," Proc. IEEE International Conference on Image Processing WA-L2.6, $1-5$ (2017).

[9] Goodman, J. W., [Introduction to Fourier optics], McGraw-Hill, San Francisco (1968).

[10] Ruderman, D. L. and Bialek, W., "Statistics of natural images: Scaling in the woods," Phys. Rev. Letters 73(6), 814-817 (1994).

[11] Clark, R. N., "Digital camera reviews and sensor performance summary." http://www.clarkvision. com/articles/digital.sensor.performance.summary/. Last Update: 2016-10-14.

[12] Stork, D. G. and Robinson, M. D., "Theoretical foundations for joint digital-optical analysis of electrooptical imaging systems," Applied Optics 47(10), B64-B75 (2008).

[13] Vettenburg, T., N. Bustin, D., and A.R., H., "Fidelity optimization for aberration-tolerant hybrid imaging systems," Optics Express 18(9), 9220-9228 (2010).

[14] Portilla, J., Tristan-Vega, A., and Selesnick, I., "Efficient and robust image restoration using multiplefeature L2-relaxed sparse analysis priors," IEEE Transactions on Image Processing 24, 5046-5059 (Dec 2015). @Matlab code publicly available at DOI: 10.13140/RG.2.1.3531.5280. 